\title{
ACCURATE DIFFERENCE METHODS FOR LINEAR ORDINARY DIFFERENTIAL SYSTEMS SUBJECT TO LINEAR CONSTRAINTS*
}

\author{
HERBERT B. KELLER $†$
}

1. Introduction. We consider the general system of $n$ first order linear ordinary differential equations

$$
\mathbf{y}^{\prime}(t)=A(t) \mathbf{y}(t)+\mathbf{g}(t),
$$$$
a<t<b,
$$

subject to $n$ "boundary" conditions, or rather $n$ linear constraints, of the form

$$
\sum_{v=1}^{N} B_{v} \mathbf{y}\left(\tau_{v}\right)=\boldsymbol{\beta}
$$

Here $\mathbf{y}(t), \mathbf{g}(t)$ and $\boldsymbol{\beta}$ are $n$-vectors and $A(t), B_{1}, \cdots, B_{N}$ are $n \times n$ matrices. The $N$ distinct points $\left\{\tau_{v}\right\}$ lie in $[a, b]$ and we only require $N \geqq 1$. Thus as special cases initial value problems, $N=1$, are included as well as the general 2-point boundary value problem, $N=2$, with $\tau_{1}=a, \tau_{2}=b$. (More general linear constraints are also studied, see (5.1) and (5.17).)

To include many other important practical applications we allow the coefficients $A(t)$ and inhomogeneous data $\mathbf{g}(t)$ to be piecewise smooth. More precisely we say that a function $\phi(t) \in P C_{m}[a, b]$ if and only if $\phi(t), \phi^{(1)}(t), \cdots, \phi^{(m)}(t)$ are piecewise continuous on $[a, b]$ with at most a finite number of jump discontinuities there. Thus left and right limits exist at the points of discontinuity. In any particular problem the fixed finite set of points of discontinuity will be assumed to be a subset of the points $\left\{\tau_{v}\right\}$ employed in (1.2). Of course if such a point is not intended to enter as a "boundary" point we simply set the corresponding $B_{v} \equiv 0$. This is strictly a device of notational convenience. We shall say that a matrix $A(t) \in P C_{m}[a, b]$ if and only if this is true for each of its components.

In $\S 2$ we show that a unique continuous solution, with piecewise smooth derivatives, of the problem (1.1)-(1.2) exists if and only if a specific matrix is nonsingular. Then we present some sufficient conditions, which may be of practical utility, to insure the nonsingularity of this matrix. In $\S 3$ a very simple difference scheme approximating $(1.1),(1.2)$ on a nonuniform net is shown to have a solution, given explicitly, whenever the boundary value problem has a unique solution and the mesh is sufficiently fine. This scheme furnishes $O\left(h^{2}\right)$ accurate numerical solutions if $A(t)$ and $\mathbf{g}(t) \in P C_{2}[a, b]$. We show, in $\S 4$, that $O\left(h^{2 M+1}\right)$ accuracy can be obtained by $h \rightarrow 0$ extrapolation if $A(t)$ and $\mathbf{g}(t) \in P C_{2 M+1}[a, b]$.

\footnotetext{
* Received by the editors August 12, 1968.

+ Applied Mathematics, Firestone Laboratories, California Institute of Technology, Pasadena. California 91109. This work was supported by the U.S. Army Research Office, Durham, under Contract DAHC 04-68-C-0006.
} 
In $\S 5$ we show how all of the above results apply to more general (integral) boundary conditions or constraints. Most of our results go over without difficulty to nonlinear systems of differential equations and even nonlinear boundary conditions. However we only present the linear theory here as it forms the basis for these other applications and is of independent interest. An account of some of the current work is contained in [7].

High order accurate approximations to solutions of boundary value problems with only piecewise smooth data and solutions do not seem to have been previously considered. However such problems occur frequently, and so we briefly discuss their formulation in the form (1.1), (1.2). The simplest and most familiar example consists in a self-adjoint equation, say

$$
\left(p(x) u_{x}\right)_{x}-q(x) u=f(x),
$$

where $p(x)$ has a point of discontinuity at which it is required that $p(x) u^{\prime}(x)$ be continuous. To replace this equation by a first order system we introduce the variables

$$
\mathbf{y}(x) \equiv\left(\begin{array}{l}
y_{1}(x) \\
y_{2}(x)
\end{array}\right) \equiv\left(\begin{array}{c}
u(x) \\
p(x) u^{\prime}(x)
\end{array}\right)
$$

to obtain

$$
\mathbf{y}^{\prime}=\left(\begin{array}{cc}
0 & 1 / p(x) \\
q(x) & 0
\end{array}\right) \mathbf{y}+\left(\begin{array}{c}
0 \\
f(x)
\end{array}\right)
$$

The jump condition is now insured by simply requiring $\mathbf{y}(x)$ to be continuous.

Unfortunately the above device is not always applicable. To illustrate the general case we consider a 2-point boundary value problem for a first order system of $n$-equations :

$$
\mathbf{u}^{\prime}=P(t) \mathbf{u}+\mathbf{q}(t), \quad D_{1} \mathbf{u}(a)+D_{2} \mathbf{u}(b)=\alpha,
$$

subject to the jump condition, at some point $c \in(a, b)$,

$$
E_{1} \mathbf{u}(c-0)+E_{2} \mathbf{u}(c+0)=\gamma .
$$

Here $D_{v}, E_{v}, P(t)$ are $n \times n$ matrices, $\mathbf{u}(t), \mathbf{q}(t), \boldsymbol{\alpha}, \boldsymbol{\gamma}$ are $n$-vectors. The idea now is to consider two systems of differential equations of the above form, say one for $\mathbf{v}(t)$ on $[a, c]$, the other for $\mathbf{w}(t)$ on $(c, b]$, and to determine conditions such that with $\mathbf{u}(t) \equiv \mathbf{v}(t)$ on $[a, c)$ and $\mathbf{u}(t) \equiv \mathbf{w}(t)$ on $(c, b]$ the function $\mathbf{u}(t)$ satisfies (1.3). The simplest way to formulate such a procedure is to extend each subinterval to $[a, b]$ and introduce the $2 n$-dimensional vectors and $2 n \times n$ order matrices

$$
\begin{aligned}
\mathbf{y}(t) & \equiv\left(\begin{array}{c}
\mathbf{v}(t) \\
\mathbf{w}(t)
\end{array}\right), \quad \mathbf{g}(t) \equiv\left(\begin{array}{l}
\mathbf{q}(t) \\
\mathbf{q}(t)
\end{array}\right), \quad \boldsymbol{\beta} \equiv\left(\begin{array}{l}
\alpha \\
\gamma
\end{array}\right), \quad A(t) \equiv\left(\begin{array}{cc}
P(t) & 0 \\
0 & P(t)
\end{array}\right), \\
B_{1} & \equiv\left(\begin{array}{cc}
D_{1} & 0 \\
0 & 0
\end{array}\right), \quad B_{2} \equiv\left(\begin{array}{cc}
0 & 0 \\
E_{1} & E_{2}
\end{array}\right), \quad B_{3} \equiv\left(\begin{array}{cc}
0 & D_{2} \\
0 & 0
\end{array}\right) .
\end{aligned}
$$


Then (1.3) is equivalent to (1.1)-(1.2) with $N=3, \tau_{1}=a, \tau_{2}=c, \tau_{3}=b$. Of course the values of $\mathbf{v}(t)(\mathbf{w}(t))$ are not required on $(c, b]([a, c))$ and this can simplify some of the work in carrying out the numerical solution.

Another, perhaps preferable, procedure is to introduce a new independent variable $s$ on each subinterval by

$$
s=\frac{t-a}{c-a}, \quad t \in[a, c], \quad s=\frac{t-c}{b-c}, \quad t \in[c, b] .
$$

Now for $s \in[0,1]$ we introduce the vectors and matrices

$$
\begin{aligned}
\mathbf{y}(s) & \equiv\left(\begin{array}{c}
\mathbf{v}(s) \\
\mathbf{w}(s)
\end{array}\right), \quad \mathbf{g}(s) \equiv\left(\begin{array}{l}
\mathbf{q}([c-a] s+a) \\
\mathbf{q}([b-c] s+c)
\end{array}\right), \quad \boldsymbol{\beta} \equiv\left(\begin{array}{l}
\alpha \\
\gamma
\end{array}\right), \\
A(s) & =\left(\begin{array}{cc}
P([c-a] s+a) & 0 \\
0 & P([b-c] s+c)
\end{array}\right), \quad B_{1} \equiv\left(\begin{array}{cc}
D_{1} & 0 \\
0 & E_{1}
\end{array}\right), \\
B_{2} & \equiv\left(\begin{array}{cc}
0 & D_{2} \\
E_{2} & 0
\end{array}\right) .
\end{aligned}
$$

Then problem (1.1)-(1.2) with $t \equiv s$ on $[a, b] \equiv[0,1]$ and $N=2, \tau_{1}=0, \tau_{2}=1$ is equivalent to (1.3). The solution of $(1.3)$ is given in terms of $y(s)$, the solution of (1.1)-(1.2) as

$$
\mathbf{u}(t)=\mathbf{v}\left(\frac{t-a}{c-a}\right), \quad t \in[a, c] ; \quad \mathbf{u}(t)=\mathbf{w}\left(\frac{t-c}{b-c}\right), \quad t \in[c, b] .
$$

Our rescaling procedure does not introduce any new "boundary" points, but it again doubles the order of the system to be solved. However, since $A(s)$ is again block diagonal, the numerical computations simplify, for the most part, as in the treatment of systems of only $n$ equations. The generalization to more than one point of discontinuity is clear. It should be mentioned that this device is quite useful in treating delay-differential equation problems, but we do not go into the details of such applications here.

2. Existence theory. The existence and uniqueness theory for linear multipoint boundary value problems of the form (1.1)-(1.2) is more or less standard. We first present the basic result in the form most useful for our work. Then we present a sequence of sufficient conditions which are more practical for verification in particular cases.

TheOREM 2.1. Let $A(t) \in P C_{m}[a, b]$ for some integer $m \geqq 0$. For any $\tau_{0} \in[a, b]$ define the (n-th order matrix) fundamental solution $Y\left(t, \tau_{0}\right)$ by

$$
Y^{\prime}=A(t) Y, \quad Y\left(\tau_{0}, \tau_{0}\right)=I .
$$


Then for each $\mathbf{g}(t) \in P C_{m}[a, b]$ and $\boldsymbol{\beta}$ the problem (1.1)-(1.2) has a unique continuous solution $y(t) \in P C_{m+1}[a, b]$ if and only if the $n$-th order matrix $Q$, defined by

$$
Q \equiv \sum_{v=1}^{N} B_{v} Y\left(\tau_{v}, \tau_{0}\right)
$$

is nonsingular.

Proof. By the standard uniqueness theorem for linear initial value problems and the variation of parameters formula it follows that a vector $\mathbf{y}(t) \in C[a, b]$, which takes on the value $\mathbf{y}\left(\tau_{0}\right)=\xi$, is a solution of the differential equation (1.1) if and only if

$$
\mathbf{y}(t)=Y\left(t, \tau_{0}\right) \xi+\int_{\tau_{0}}^{t} Y(t, \tau) \mathbf{g}(\tau) d \tau
$$

However this function satisfies the boundary conditions (1.2) if and only if

$$
Q \xi=\boldsymbol{\beta}-\sum_{v=1}^{N} B_{v} \int_{\tau_{0}}^{\tau_{v}} Y\left(\tau_{v}, \tau\right) \mathbf{g}(\tau) d \tau
$$

Thus it follows that problem (1.1) (1.2) has a unique continuous solution for each $\boldsymbol{\beta}$ and $\mathbf{g}(t)$ if and only if the linear system (2.4) has a unique solution $\xi$ for each $\boldsymbol{\beta}$ and $\mathbf{g}(t)$. But this is just equivalent to the nonsingularity of $Q$.

Finally we observe that if (1.1) has a continuous solution, then, by differentiation of the equation, this solution has one more piecewise continuous derivative on $[a, b]$ than do the data $A(t)$ and $\mathbf{g}(t)$. This completes the proof.

An equivalent, perhaps more familiar, formulation of the above necessary and sufficient condition is that the homogeneous problem corresponding to (1.1) $-(1.2)$ has only the trivial solution $\mathbf{y}(t) \equiv 0$. Clearly this yields, from (2.4) with $\boldsymbol{\beta} \equiv \mathbf{g}(t) \equiv 0$, the homogeneous linear system $Q \xi=0$, and the same result follows. In this form the result is well known for the case $N=2$; see [1, pp. 295].

The statement in Theorem 2.1 yields, in principle, a specific test for the unique solvability of the boundary value problem. However it is usually not practical to determine the nonsingularity of the matrix $Q$ directly. Thus we turn to the consideration of sufficient conditions which are more easily applied. For example, if the matrix $Q_{0} \equiv \sum_{v=1}^{N} B_{v}$ is nonsingular, then we claim that $Q$ is nonsingular provided $|b-a|$ is sufficiently small. In fact we define a sequence of matrices $\left\{Q_{m}\right\}$ which are such that if $Q_{M}$ is nonsingular and $|b-a|$ is sufficiently small then so are $Q_{m}$ for all $m>M$ and $Q=\lim _{m \rightarrow \infty} Q_{m}$ is also nonsingular. The allowed interval lengths $|b-a|$ increase with $m$. Specifically we have the following theorem.

THEOREM 2.2. Let $A(t) \in P C_{0}[a, b]$ and define, for each $m=0,1,2, \cdots$, the matrix

$$
\begin{gathered}
Q_{m} \equiv \sum_{v=1}^{N} B_{v} Y_{m}\left(\tau_{v}, \tau_{0}\right) \\
Y_{0}\left(\tau, \tau_{0}\right) \equiv I, \quad Y_{m}\left(\tau, \tau_{0}\right)=I+\int_{\tau_{0}}^{\tau} A(s) Y_{m-1}\left(s, \tau_{0}\right) d s, \quad m=1,2, \cdots
\end{gathered}
$$


Define $k(t) \equiv\|A(t)\|$. For some $\lambda$ in $0 \leqq \lambda<1$ and integer $M \geqq 0$ let $Q_{M}$ be nonsingular and satisfy

$$
\begin{aligned}
& \sum_{v=1}^{N}\left\|Q_{M}^{-1} B_{v}\right\|\left\{\exp \left(\left|\int_{\tau_{0}}^{\tau_{v}} k(s) d s\right|\right)\right. \\
& \left.\quad-\left[1+\left|\int_{\tau_{0}}^{\tau_{v}} k(s) d s\right|+\cdots+\frac{1}{M !}\left|\int_{\tau_{0}}^{\tau_{v}} k(s) d s\right|\right]\right\} \leqq \lambda .
\end{aligned}
$$

Then the matrices $Q_{m}$ for all $m>M$ and the matrix $Q$ defined by (2.1)-(2.2) are nonsingular.

$$
\text { Let } \tau_{0}=(a+b) / 2, K \equiv \max _{a \leqq t \leqq b} k(t) \text { and suppose } Q_{M} \text { satisfies }
$$

$$
\frac{1}{(M+1) !}\left(\frac{K|b-a|}{2}\right)^{M+1} e^{K|b-a| / 2} \leqq \frac{\lambda}{\sum_{v=1}^{N}\left\|Q_{M}^{-1} B_{v}\right\|} .
$$

Then (2.6) is satisfied.

Proof. Since $Q_{M}$ is nonsingular, by hypothesis, we can write $Q_{M+r}$ for any $r=1,2, \cdots$ as

$$
Q_{M+r}=Q_{M}\left\{I+\sum_{v=1}^{N} Q_{M}^{-1} B_{v}\left[Y_{M+r}\left(\tau_{v}, \tau_{0}\right)-Y_{M}\left(\tau_{v}, \tau_{0}\right)\right]\right\} .
$$

From the definition $(2.5 b)$ we get

$$
\begin{aligned}
\left\|Y_{M+r}(\tau, t)-Y_{M}(\tau, t)\right\| & \leqq \sum_{\mu=M+1}^{M+r}\left|\int_{t}^{\tau} k\left(t_{1}\right) \int_{t}^{t_{1}} k\left(t_{2}\right) \cdots \int_{t}^{t_{\mu-1}} k\left(t_{\mu}\right) d t_{\mu} \cdots d t_{i}\right| \\
& \leqq \sum_{\mu=M+1}^{\infty} \frac{1}{\mu !}\left|\int_{t}^{\tau} k(\xi) d \xi\right|^{\mu} \\
& =\exp \left(\left|\int_{t}^{\tau} k(\xi) d \xi\right|\right)-\sum_{\mu=0}^{M} \frac{1}{\mu !}\left|\int_{t}^{\tau} k(\xi) d \xi\right|^{\mu} .
\end{aligned}
$$

The final right-hand side above is independent of $r$ and since the fundamental solution $Y(\tau, t)$ has the convergent series representation (i.e., the Matrizant, see $[5$, pp. 408-411])

$$
Y(\tau, t)=\lim _{m \rightarrow \infty} Y_{m}(\tau, t),
$$

we obtain, on letting $r \rightarrow \infty$ in (2.9a),

$$
\left\|Y(\tau, t)-Y_{M}(\tau, t)\right\| \leqq \exp \left(\left|\int_{t}^{\tau} k(\xi) d \xi\right|\right)-\sum_{\mu=0}^{M} \frac{1}{\mu !}\left|\int_{t}^{\tau} k(\xi) d \xi\right|^{\mu} .
$$

Using (2.9) and (2.6) we obtain

$$
\left\|\sum_{v=1}^{N} Q_{M}^{-1} B_{v}\left[Y_{M+r}\left(\tau_{v}, \tau_{0}\right)-Y_{M}\left(\tau_{v}, \tau_{0}\right)\right]\right\| \leqq \lambda
$$


and

$$
\left\|\sum_{v=1}^{N} Q_{M}^{-1} B_{v}\left[Y\left(\tau_{v}, \tau_{0}\right)-Y_{M}\left(\tau_{v}, \tau_{0}\right)\right]\right\| \leqq \lambda .
$$

Since $\lambda<1$ and $Q_{M}$ is nonsingular we conclude from (2.8) and the Banach lemma that $Q_{M+r}$ is nonsingular for all $r=1,2, \cdots$. Similarly from (2.2) and the above it follows that $Q$ is nonsingular.

Finally the inequality (2.7) implies (2.6) since $\left|\tau_{v}-\tau_{0}\right| \leqq|b-a| / 2$ when $\tau_{0}=(a+b) / 2$,

$$
\left|\int_{\tau_{0}}^{\tau_{\nu}} k(s) d s\right| \leqq \frac{K|b-a|}{2}
$$

and for any $z \geqq 0$,

$$
e^{z}-\left[1+z+\cdots+\frac{z^{M}}{M !}\right] \leqq \frac{z^{M+1}}{(M+1) !} e^{z}
$$

This completes the proof.

It should be observed that the choice $\tau_{0}=\tau_{v}$ for some particular $v>0$ results in the elimination of the term containing the factor $\left\|Q_{M}^{-1} B_{v}\right\|$ from the sum in (2.8). Thus, in particular, for two-point boundary value problems, $N=2$, we find that (2.6) is satisfied if

$$
\frac{1}{(M+1) !}(K|b-a|)^{M+1} e^{K|b-a|} \leqq \frac{\lambda}{\theta}, \quad \theta \equiv \min \left(\left\|Q_{M}^{-1} B_{1}\right\|,\left\|Q_{M}^{-1} B_{2}\right\|\right)
$$

and $\tau_{0}$ has the appropriate value $\tau_{0}=a$ or $\tau_{0}=b$. For pure initial value problems, $N=1$, the condition is automatically satisfied by the obvious choice $\tau_{0}=\tau_{1}$.

As an example of the use and limitations of Theorem 2.2 we consider briefly the general second order two-point boundary value problem with separated (or "unmixed") end conditions :

$$
\begin{gathered}
\phi^{\prime \prime}=p(t) \phi^{\prime}+q(t) \phi+r(t), \\
a_{0} \phi(a)+a_{1} \phi^{\prime}(a)=a_{2}, \quad b_{0} \phi(b)+b_{1} \phi^{\prime}(b)=b_{2} .
\end{gathered}
$$

An equivalent first order system of the form (1.1)-(1.2) is obtained by means of the definitions:

$$
\begin{aligned}
& \mathbf{y} \equiv\left(\begin{array}{l}
\phi \\
\phi^{\prime}
\end{array}\right), \quad A(t) \equiv\left(\begin{array}{cc}
0 & 1 \\
p(t) & q(t)
\end{array}\right), \quad \mathbf{g}(t) \equiv\left(\begin{array}{c}
0 \\
r(t)
\end{array}\right), \\
& B_{1} \equiv\left(\begin{array}{cc}
a_{0} & a_{1} \\
0 & 0
\end{array}\right), \quad B_{2} \equiv\left(\begin{array}{cc}
0 & 0 \\
b_{0} & b_{1}
\end{array}\right), \quad \boldsymbol{\beta} \equiv\left(\begin{array}{l}
a_{2} \\
b_{2}
\end{array}\right) .
\end{aligned}
$$

From (2.5) we find that in this case

$$
Q_{0}=\left(\begin{array}{ll}
a_{0} & a_{1} \\
b_{0} & b_{1}
\end{array}\right)
$$


and hence it is nonsingular if and only if $a_{0} b_{1} \neq a_{1} b_{0}$. Thus, for instance, if $\phi$ (or $\phi^{\prime}$ ) is specified at both endpoints, $Q_{0}$ is singular. (In general $Q_{0}$ is nonsingular if the boundary conditions are linearly independent when applied at the same point.) In the next order approximation we find with $\tau_{0}=\tau_{1}=a$ and $\tau_{2}=b$ that

$$
Q_{1}=\left(\begin{array}{cc}
a_{0} & a_{1} \\
b_{0}+b_{1} \int_{a}^{b} p(t) d t & b_{0}(b-a)+b_{1}\left[1+\int_{a}^{b} q(t) d t\right]
\end{array}\right) .
$$

Thus

$$
\operatorname{det} Q_{1}=\operatorname{det} Q_{0}+a_{0} b_{0}(b-a)+a_{0} b_{1} \int_{a}^{b} q(t) d t-a_{1} b_{1} \int_{a}^{b} p(t) d t,
$$

and clearly $Q_{1}$ may be nonsingular when $\operatorname{det} Q_{0}=0$. In fact with $a_{1}=b_{1}=0$, $a_{0} b_{0} \neq 0$, which corresponds to specifying $\phi$ at the endpoints, $Q_{1}$ is nonsingular. Then condition (2.10) becomes

$$
\frac{K^{2}|b-a|}{2} e^{K|b-a|} \leqq \lambda<1,
$$

where $K \equiv \max _{a \leqq t \leqq b}(|p(t)|+|q(t)|, 1)$. Of course the problem (2.11) has a unique solution under much weaker conditions than the above.

Finally we point out that the least restrictions we can impose on the matrices $B_{v}$ in order to maintain uniqueness of solutions of (1.1)-(1.2) is that the $n$ boundary conditions in (1.2) should be linearly independent. Their independence is easily shown to be equivalent to the assertion that

$$
\operatorname{rank}\left(B_{1}, B_{2}, \cdots, B_{N}\right)=n,
$$

where $\left(B_{1}, B_{2}, \cdots, B_{N}\right)$ is the $n \times n N$ order matrix whose columns are those of the $B_{v}$. However a matrix of this form has rank $n$ if and only if there exist $n$th order matrices $C_{v}$ such that $\sum_{v=1}^{N} B_{v} C_{v}$ is nonsingular. Thus from Theorem 2.1 it follows that the above independence condition is necessary for uniqueness.

3. Finite difference approximations. On the interval $[a, b]$ we place a net of points $\left\{t_{j}\right\}$ with

$$
t_{0}=a, \quad t_{j}=t_{j-1}+h_{j}, \quad j=1,2, \cdots, J, \quad t_{J}=b .
$$

The mesh widths $h_{j}$ are to be chosen so that all the special points $\tau_{v}, v=1,2, \cdots, N$, at which the data $A(t)$ and $\mathbf{g}(t)$ or their derivatives have jump discontinuities or at which "boundary" conditions are imposed are points of the net. Thus $J \geqq N$ and in particular for any such net we denote these special net points as

$$
t_{j_{v}}=\tau_{v}, \quad v=1,2, \cdots, N .
$$

Our analysis usually refers to a family of such nets in which $J \rightarrow \infty$ while $\max _{j} h_{j} \rightarrow 0$. For each net in any such family we further require that for some 
fixed positive number $\lambda \geqq 1$,

$$
h \equiv \max _{j} h_{j} \leqq \lambda \min _{k} h_{k}
$$

At each point $t_{j}$ of a fixed net we seek a vector $\mathbf{u}_{j}$ which is to approximate $\mathbf{y}\left(t_{j}\right)$, the solution of (1.1), (1.2). The approximating net function $\left\{\mathbf{u}_{j}\right\}$ is defined by the finite difference equations

$$
L_{h} \mathbf{u}_{j} \equiv h_{j}^{-1}\left(\mathbf{u}_{j}-\mathbf{u}_{j-1}\right)-\frac{1}{2} A\left(t_{j-1 / 2}\right)\left(\mathbf{u}_{j}+\mathbf{u}_{j-1}\right)=\mathbf{g}\left(t_{j-1 / 2}\right), j=1,2, \cdots, J,
$$

and the boundary conditions

$$
\sum_{v=1}^{N} B_{v} \mathbf{u}_{j_{v}}=\boldsymbol{\beta}
$$

Here we have used the notation

$$
t_{j-1 / 2} \equiv t_{j}-\frac{1}{2} h_{j}=t_{j-1}+\frac{1}{2} h_{j}, \quad j=1,2, \cdots, J .
$$

The difference scheme in (3.2) is frequently called the "centered difference" method or, in analogy with quadrature schemes, we shall call it the "midpoint rule." An obvious alternative would seem to be the "centered Euler" scheme, analogous to the trapezoidal rule, in which the zero order term is approximated by

$$
\frac{1}{2}\left[A\left(t_{j}\right) \mathbf{u}_{j}+A\left(t_{j-1}\right) \mathbf{u}_{j-1}\right]
$$

However we do not employ this choice, as the treatment of discontinuous coefficients then requires special care.

The stability of the midpoint rule for initial value problems is straightforward. But since this result is basic for our work we present it in the following lemma.

LEMMA 3.1. Let $K \equiv \max _{a \leqq t \leqq b}\|A(t)\|$. Then for any net (3.1) with $h \leqq h_{0}<2 / K$ and for any net function $\left\{\phi_{j}\right\}$ on this net,

$$
\left\|\boldsymbol{\phi}_{j}\right\| \leqq e^{\lambda K^{*}\left|t_{j}-t_{0}\right|}\left\{\left\|\boldsymbol{\phi}_{0}\right\|+\frac{2}{K} \max _{1 \leqq i \leqq J}\left\|L_{h} \boldsymbol{\phi}_{i}\right\|\right\}, \quad j=0,1, \cdots, J,
$$

where

$$
K^{*} \equiv \frac{K}{1-h_{0} K / 2}
$$

Proof. It follows from the hypothesis and the Banach lemma that the matrices

$$
R_{j} \equiv I-\frac{1}{2} h_{j} A\left(t_{j-1 / 2}\right), \quad j=1,2, \cdots, J,
$$

are nonsingular. Then from the definition (3.2) of $L_{h}$ and with

$$
P_{j} \equiv\left[I-\frac{1}{2} h_{j} A\left(t_{j-1 / 2}\right)\right]^{-1}\left[I+\frac{1}{2} h_{j} A\left(t_{j-1 / 2}\right)\right], \quad j=1,2, \cdots, J,
$$


we have the identities

$$
\boldsymbol{\phi}_{j} \equiv P_{j} \phi_{j-1}+h_{j} R_{j}^{-1} L_{h} \phi_{j}, \quad j=1,2, \cdots, J .
$$

By induction this yields

$$
\begin{aligned}
\phi_{j} \equiv & \left(P_{j} P_{j-1} \cdots P_{1}\right) \phi_{0} \\
& +\left\{h_{j} R_{j}^{-1} L_{h} \phi_{j}+\sum_{i=1}^{j-1}\left(P_{j} P_{j-1} \cdots P_{i+1}\right) h_{i} R_{i}^{-1} L_{h} \phi_{i}\right\}, j=1,2, \cdots, J .
\end{aligned}
$$

Taking norms above and employing the inequalities, valid for $h \leqq h_{0}$,

$$
\left\|R_{j}^{-1}\right\| \leqq \frac{1}{1-h K / 2}, \quad\left\|P_{j}\right\| \leqq \frac{1+h K / 2}{1-h K / 2} \leqq e^{h K^{*}}
$$

we sum the resulting geometric progression and obtain (3.4) since

$$
j h \leqq \lambda \sum_{i=1}^{j} h_{i}=\lambda\left|t_{j}-t_{0}\right|
$$

This completes the proof.

The solution to the finite difference boundary value problem (3.2)-(3.3) can be given explicitly for sufficiently small net spacing. This is most easily done by factoring the coefficient matrix into a product of block upper triangular by lower triangular matrices. The details and justification are contained in the proof of the following lemma.

Lemma 3.2. Define the $n$-th order matrices $C_{j}$ and $S_{j}$ by:

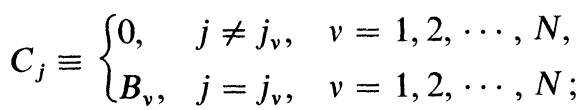

$$
\begin{aligned}
& S_{J} \equiv C_{J}, \quad S_{j-1}=C_{j-1}+S_{j} P_{j}, \quad j=J, J-1, \cdots, 1 .
\end{aligned}
$$

Then if $A(t) \in P C_{0}[a, b]$ and the boundary value problem (1.1)-(1.2) has a unique solution, the matrix $S_{0}$ is nonsingular for $h$ sufficiently small. In this case the finite difference boundary value problem (3.2)-(3.3) also has a unique solution and it is given by

$$
\begin{aligned}
& \mathbf{u}_{0}=S_{0}^{-1}\left[\boldsymbol{\beta}-\sum_{j=1}^{J} h_{j} S_{j} R_{j}^{-1} \mathbf{g}\left(t_{j-1 / 2}\right)\right], \\
& \mathbf{u}_{j}=P_{j} \mathbf{u}_{j-1}+h_{j} R_{j}^{-1} \mathbf{g}\left(t_{j-1 / 2}\right), \quad j=1,2, \cdots, J .
\end{aligned}
$$

Proof. Using (3.5) we can write (3.2) in an equivalent form which is just (3.8b). Then the coefficient matrix of the system consisting of (3.3) and (3.8b) 
can be written in block form and factored, using the definitions (3.7), as follows:

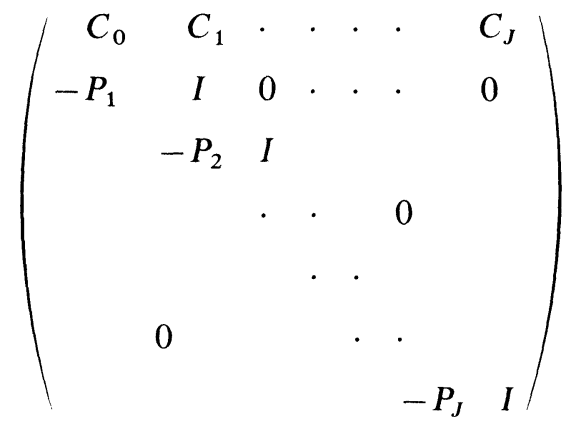

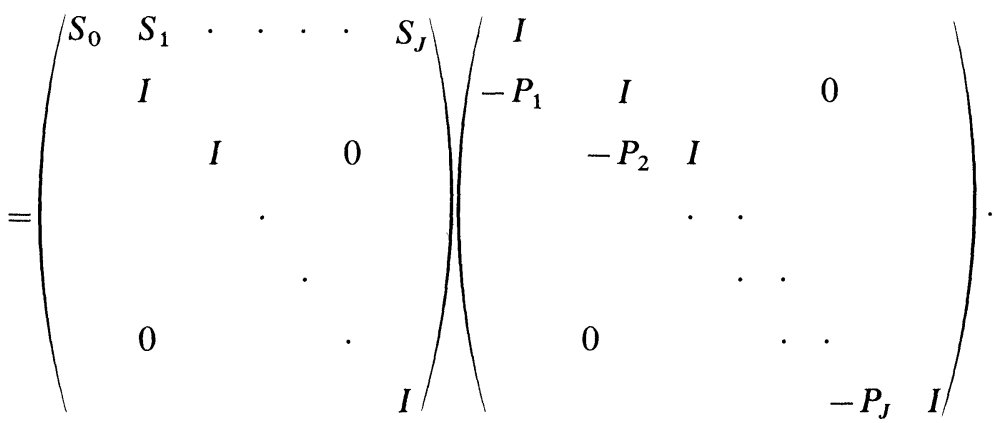

The solvability of the system (3.2)-(3.3) and the explicit form of the solution given in (3.8) clearly follow from the above factorization if the matrix $S_{0}$ is nonsingular.

We can write the matrix $S_{0}$ in the form

$$
S_{0}=\sum_{j=0}^{J} C_{j} Z_{j},
$$

where the matrices $Z_{j}$ are defined by the recursions

$$
Z_{0}=I, \quad Z_{j}=P_{j} Z_{j-1},
$$$$
j=1,2, \cdots, J \text {. }
$$

Note, by (3.5), that $Z_{j}$ is the solution of the difference problem

$$
L_{h} Z_{j}=0, \quad j=1,2, \cdots, J, \quad Z_{0}=I .
$$

Thus the $Z_{j}$ may be approximations on the net to the fundamental solution

$$
Z^{\prime}(t)=A(t) Z(t), \quad Z(a)=I .
$$

Since $A(t) \in P C_{0}[a, b]$ it follows that $Z(t) \in P C_{1}[a, b]$ and satisfies

$$
L_{h} Z\left(t_{j}\right)=\sigma_{j}, j=1,2, \cdots, J, \quad Z\left(t_{0}\right)=I .
$$

Here $\sigma_{j}$ are the local truncation errors and $\left\|\sigma_{j}\right\|=O\left(\omega_{1}(h / 2)\right)$, where $\omega_{1}(\delta)$ is the modulus of continuity of $Z^{\prime}(t)$ over any interval of continuity on $[a, b]$. From (3.11a), (3.11c) and Lemma 3.1, assuming $h<2 / K$, we obtain

$$
\left\|Z\left(t_{j}\right)-Z_{j}\right\| \leqq O\left(\left\|\sigma_{j}\right\|\right), \quad j=1, \cdots, J .
$$


Since $Z(t)$ and $Y\left(t, \tau_{0}\right)$ defined in (2.1) are fundamental solutions of the same system, it follows that

$$
Z(t)=Y\left(t, \tau_{0}\right) Y^{-1}\left(t_{0}, \tau_{0}\right)
$$

and thus from the above estimate

$$
Z_{j}=Y\left(t_{j}, \tau_{0}\right) Y^{-1}\left(t_{0}, \tau_{0}\right)+O\left(\sigma_{j}\right)
$$

Recalling the definitions in (3.7) and (2.2) we find that

$$
S_{0}=Q Y^{-1}\left(t_{0}, \tau_{0}\right)+O\left(\sum_{j=1}^{J} C_{j} \sigma_{j}\right) .
$$

But by Theorem 2.1 the matrix $Q$ is nonsingular since (1.1)-(1.2) is assumed to have a unique solution. Further since at most $N$ (independent of $J$ ) of the matrices $C_{j}$ are nonzero and the local truncation errors $\sigma_{j}$ can be made arbitrarily small as $h \rightarrow 0$, it follows from the Banach lemma that $S_{0}$ is nonsingular for sufficiently small $h$. This completes the proof.

It should be noted that the terms $O\left(\omega_{1}(h / 2)\right)$ in the above proof can be replaced by terms which are $O\left(h^{2}\right)$ if $A(t) \in P C_{2}[a, b]$. The formulas (3.8) for the solution of the difference problem may not be the most appropriate to employ in actual calculations. Specifically suppose $N=2$ and the two-point conditions are not mixed, a very common case. Then, by placing those conditions in (3.3) that occur at $\tau_{1}=a$ first and those at $\tau_{2}=b$ last, the coefficient matrix of the system replacing (3.2)-(3.3) becomes a band matrix (that is with all nonzero elements clustered about the diagonal). The resulting system can frequently be solved by the familiar band-matrix factoring procedure which is actually Gauss elimination accounting for zero elements. Note that this is not the procedure employed in (3.9). In fact, when applicable, the present procedure may be somewhat more accurate than that in (3.9) as regards the growth of roundoff and possible loss in accuracy due to cancellation of leading digits. Of course, for exact arithmetic there is no difference between the two procedures. (In evaluating $S_{0}$ and $\mathbf{u}_{0}$ from (3.7) and (3.8) the inverses $R_{j}^{--1}$ should usually be avoided and the corresponding systems of order $n$ solved by elimination instead.)

We may now employ the solution algorithm in Lemma 3.2 to demonstrate the stability of the centered difference boundary value scheme (3.2)-(3.3). This is to be contrasted to the stability result in Lemma 3.1 for the centered difference initial value scheme (3.2) with $\mathbf{u}_{0}$ given.

Lemma 3.3. Let $A(t) \in P C_{0}[a, b]$, define $K \equiv \max _{a \leqq t \leqq b}\|A(t)\|$ and let $h$ be so small that Lemmas 3.1-3.2 hold. Then there exist constants $h_{0}, K_{1}$ and $K_{2}$ independent of $J$ such that on all nets (3.1) with $h \leqq h_{0}$ any net function $\left\{\boldsymbol{\phi}_{j}\right\}$ satisfies

$$
\left\|\boldsymbol{\phi}_{j}\right\| \leqq K_{1}\left\|\sum_{v=1}^{N} B_{v} \boldsymbol{\phi}_{v}\right\|+K_{2} \max _{1 \leqq i \leqq J}\left\|L_{h} \boldsymbol{\phi}_{i}\right\|, \quad j=0,1, \cdots, J .
$$


Proof. From the result in Lemma 3.2 and with the identity (3.6a) we have the identity

and thus

$$
\phi_{0}=S_{0}^{-1}\left[\sum_{v=1}^{N} B_{v} \phi_{v}-\sum_{j=1}^{J} h_{j} S_{j} R_{j}^{-1} L_{h} \phi_{j}\right]
$$

$$
\left\|\phi_{0}\right\| \leqq\left\|S_{0}^{-1}\right\|\left[\left\|\sum_{\nu=1}^{N} B_{v} \phi_{\nu}\right\|+\frac{b-a}{1-h K / 2} \max _{j}\left\|S_{j}\right\| \cdot\left\|L_{h} \phi_{j}\right\|\right] .
$$

It follows from (3.7b) that

$$
S_{j}=C_{j}+C_{j+1} P_{j+1}+\cdots+C_{J}\left(P_{J} P_{J-1} \cdots P_{j+1}\right), \quad j=0,1, \cdots, J-1 .
$$

Then using (3.6c) and (3.7a), we have

$$
\begin{aligned}
\left\|S_{j}\right\| & \leqq \sum_{k=j}^{J}\left\|C_{k}\right\| e^{K^{*}\left(t_{k}-t_{j}\right)} \\
& \leqq e^{K^{*}|b-a|} \sum_{v=1}^{N}\left\|B_{v}\right\| .
\end{aligned}
$$

From (3.12) we get, since $h$ is sufficiently small and $\left\|Y\left(t_{0}, \tau_{0}\right)\right\| \leqq e^{K\left|t_{0}-\tau_{0}\right|}$,

$$
\left\|S_{0}^{-1}\right\| \leqq \frac{\left\|Q^{-1}\right\| e^{K\left|t_{0}-\tau_{0}\right|}}{1-O\left(\omega_{1}(h / 2)\right)}
$$

Combining (3.14a)-(3.14c) with (3.4) we obtain (3.13) with

$$
\begin{aligned}
& K_{1}=\left\|Q^{-1}\right\| \frac{e^{K^{*}\left(|b-a|+\left|t_{0}-\tau_{0}\right|\right)}}{1-\delta_{0}}, \\
& K_{2}=e^{\lambda K^{*}|b-a|}\left[\frac{2}{K}+\frac{K_{1}|b-a|}{1-\delta_{1}} \sum_{v=1}^{N}\left\|B_{v}\right\|\right],
\end{aligned}
$$

where $\delta_{0}=1-h_{0} K / 2, K^{*}=K / \delta_{0}$ and $\delta_{1}=O\left(\omega_{1}\left(h_{0} / 2\right)\right)$. This completes the proof.

Now we present the basic convergence theorem and error estimate for the finite difference approximation to the solution of the boundary value problem.

Theorem 3.1. Let $A(t) \in P C_{p}[a, b]$ for some integer $p \geqq 0$ and be such that the multipoint boundary value problem (1.1)-(1.2) with $\mathbf{g}(t) \in C_{p}[a, b]$ has a unique solution $\mathbf{y}(t)$. Then with the constants $h_{0}, K$ and $K_{2}$ of Lemma 3.3, the solution $\left\{\mathbf{u}_{j}\right\}$ of the centered difference multipoint boundary value problem (3.2)-(3.3) satisfies, on all nets (3.1) with $h \leqq h_{0}$ :

$$
\left\|y\left(t_{j}\right)-\mathbf{u}_{j}\right\| \leqq\left\{\begin{array}{l}
K_{2}\left[\frac{M_{3}}{24}+K \frac{M_{2}}{8}\right] h^{2} \quad \text { if } p \geqq 2, \\
K_{2}\left[\frac{\omega_{2}(h)}{8}+K \frac{M_{2}}{8} h\right] h \quad \text { if } p=1, \\
K_{2}\left[\omega_{1}\left(\frac{h}{2}\right)+K \frac{\omega_{1}(h)}{4} h\right] \text { if } p=0 .
\end{array}\right.
$$


Here $M_{v}=\max _{a \leqq t \leqq b}\left\|\mathbf{y}^{(v)}(t)\right\|$ for $v=2,3$ and $\omega_{v}(\delta)$ is the modulus of continuity of $\mathbf{y}^{(v)}(t)$ on every interval of continuity in $[a, b]$.

Proof. Since $\mathbf{y}(t)$ and $\left\{\mathbf{u}_{j}\right\}$ satisfy the same boundary conditions (1.2) and (3.3), we have, on applying Lemma 3.3 to the net function $\phi_{j} \equiv \mathbf{y}\left(t_{j}\right)-\mathbf{u}_{j}$ and using (3.2) and (1.1),

$$
\begin{aligned}
&\left\|\mathbf{y}\left(t_{j}\right)-\mathbf{u}_{j}\right\| \leqq K_{2} \max _{1 \leqq i \leqq J}\left\|L_{h} \mathbf{y}\left(t_{i}\right)-\mathbf{g}\left(t_{i-1 / 2}\right)\right\| \\
& \leqq K_{2} \max _{1 \leqq i \leqq J}\left\|L_{h} \mathbf{y}\left(t_{i}\right)-L \mathbf{y}\left(t_{i-1 / 2}\right)\right\| \\
& \leqq K_{2} \max _{1 \leqq i \leqq J}\left\{\left\|\frac{\| \mathbf{y}\left(t_{i}\right)-\mathbf{y}\left(t_{i-1}\right)}{h_{i}}-\mathbf{y}^{\prime}\left(t_{i-1 / 2}\right)\right\|\right. \\
&\left.\quad+K\left\|\frac{\mathbf{y}\left(t_{i}\right)+\mathbf{y}\left(t_{i-1}\right)}{2}-\mathbf{y}\left(t_{i-1 / 2}\right)\right\|\right\} .
\end{aligned}
$$

To estimate the right-hand side above we apply Taylor's theorem on each interval $\left[t_{i-1}, t_{i}\right], i=1,2, \cdots, J$. But by the requirement (3.1b) and Theorem 2.1 it follows that $\mathbf{y}(t) \in C_{p+1}\left[t_{i-1}, t_{i}\right]$ for each $i=1,2, \cdots, J$. Thus (3.15) easily follows.

4. Higher order accurate approximations. Perhaps the simplest method for obtaining high order accurate numerical approximations is to apply Richardson's deferred approach to the limit or as it is also termed : $h \rightarrow 0$ extrapolation. Current work on such methods includes the extension of Romberg integration for nonlinear initial value problems [3] and generalizations of Fox's difference correction technique [9] for boundary value problems. The treatments to date have not noted the applicability to problems with only piecewise smooth solutions ${ }^{1}$ and have usually required uniform nets. We shall first develop the theory for uniform spacing

$$
h_{j} \equiv h \equiv \frac{b-a}{J}, \quad j=1,2, \cdots, J .
$$

This places an obvious restriction on the allowed spacing of points of discontinuity and boundary points $\left\{\tau_{v}\right\}$ which we assume to hold. Then in the next subsection we show that the severe restriction (4.1) can be removed and of course the spacing of the $\tau_{v}$ is then unrestricted.

First we present the theoretical basis for higher order accurate approximations, which is an asymptotic expansion for the error $\mathbf{u}_{j}-\mathbf{y}\left(t_{j}\right)$ in powers of $h^{2}$. Then we discuss the computation of the improved or higher order accurate solution.

THEOREM 4.1. For some integer $M \geqq 1$ let $A(t), \mathbf{g}(t) \in P C_{2 M+1}[a, b]$ and problem (1.1)-(1.2) have a unique solution. Let the boundary and discontinuity points $\left\{\tau_{v}\right\}$ satisfy (3.1) and (4.1) for some sequence of uniform nets with $J=J_{\mu} \rightarrow \infty$. For $\boldsymbol{\phi}(t) \in P C_{2 m+1}[a, b]$ define the differential $T_{m}\{\boldsymbol{\phi}(t)\} \in P C_{0}[a, b)$ by:

$$
T_{m}\{\boldsymbol{\phi}(t)\} \equiv \frac{-1}{(2 m) !}\left[\frac{1}{2 m+1} \boldsymbol{\phi}^{(2 m+1)}(t)-A(t) \boldsymbol{\phi}^{(2 m)}(t)\right], m=1,2, \cdots
$$

\footnotetext{
${ }^{1}$ For quadrature the integrand may be piecewise smooth. See $\S 5$ where this is employed.
} 
With $\mathbf{y}(t)$ the solution of (1.1)-(1.2) define the principal error functions $\mathbf{e}_{m}(t)$ as the solutions of the multipoint boundary value problems:

$$
\begin{gathered}
\mathbf{L}_{1}(t)=T_{1}\{\mathbf{y}(t)\}, \quad \sum_{v=1}^{N} B_{v} \mathbf{e}_{1}\left(\tau_{v}\right)=0 ; \\
L \mathbf{e}_{m}(t)=T_{m}\{\mathbf{y}(t)\}+\sum_{k=1}^{m-1} T_{k}\left\{\mathbf{e}_{m-k}(t)\right\}, \quad \sum_{v=1}^{N} B_{v} \mathbf{e}_{m}\left(\tau_{v}\right)=0, \\
m=2,3, \cdots, M .
\end{gathered}
$$

Then, if $\left\{\mathbf{u}_{j}\right\}$ is the solution of the finite difference boundary value problem (3.2)(3.3) on the net (3.1), (4.1),

$$
\mathbf{u}_{j}-\mathbf{y}\left(t_{j}\right)=\sum_{m=1}^{M}\left(\frac{h}{2}\right)^{2 m} \mathbf{e}_{m}\left(t_{j}\right)+O\left(h^{2 M+1}\right), \quad j=0,1, \cdots, J .
$$

Proof. By Theorem 2.1 it follows that $\mathbf{y}(t) \in P C_{2 M+2}[a, b]$. Then applying Taylor's theorem to $\mathrm{y}\left(t_{j-1 / 2} \pm h / 2\right)$ on $\left[t_{j-1}, t_{j}\right]$ we obtain, using (1.1), (3.2) and (4.2):

$$
\begin{aligned}
L_{h}\left[\mathbf{u}_{j}-\mathbf{y}\left(t_{j}\right)\right] & =L \mathbf{y}\left(t_{j-1 / 2}\right)-L_{h} \mathbf{y}\left(t_{j}\right) \\
& =\sum_{m=1}^{M}\left(\frac{h}{2}\right)^{2 m} T_{m}\left\{\mathbf{y}\left(t_{j-1 / 2}\right)\right\}+O\left(h^{2 M+1}\right), \quad j=1,2, \cdots, J .
\end{aligned}
$$

If $\phi(t) \in P C_{2 r+1}[a, b]$ and $s \leqq r$, then $T_{s}\{\phi(t)\} \in P C_{2(r-s)}[a, b]$. Thus an induction employing Theorem 2.1 yields the fact that the $\mathbf{e}_{m}(t)$ defined in (4.3) satisfy $\mathbf{e}_{m}(t) \in P C_{2(M-m)+2}[a, b]$ for $m=1,2, \cdots, M$. Again applying Taylor's theorem on $\left[t_{j-1}, t_{j}\right]$ yields as above:

$$
\begin{aligned}
L_{h} \mathbf{e}_{m}\left(t_{j}\right)=L \mathbf{e}_{m}\left(t_{j-1 / 2}\right)+\left[L_{h} \mathbf{e}_{m}\left(t_{j}\right)-L \mathbf{e}_{m}\left(t_{j-1 / 2}\right)\right] & \\
=L \mathbf{e}_{m}\left(t_{j-1 / 2}\right)-\sum_{s=1}^{M-m}\left(\frac{h}{2}\right)^{2 s} T_{s}\left\{\mathbf{e}_{m}\left(t_{j-1 / 2}\right)\right\}+O\left(h^{2(M-m)+1}\right), & \\
& m=1,2, \cdots, M-1,
\end{aligned}
$$

and

$$
L_{h} \mathbf{e}_{M}\left(t_{j}\right)=L \mathbf{e}_{M}\left(t_{j-1 / 2}\right)+O(h) .
$$

Using the differential equations in (4.3) and (4.6a), (4.6b), we obtain

$$
\begin{aligned}
\sum_{m=1}^{M}\left(\frac{h}{2}\right)^{2 m} L_{h} \mathbf{e}_{m}\left(t_{j}\right)= & \sum_{m=1}^{M}\left(\frac{h}{2}\right)^{2 m} T_{m}\left\{\mathbf{y}\left(t_{j-1 / 2}\right)\right\}+\sum_{m=2}^{M}\left(\frac{h}{2}\right)^{2 m} \sum_{k=1}^{m-1} T_{k}\left\{\mathbf{e}_{m-k}\left(t_{j-1 / 2}\right)\right\} \\
& -\sum_{m=1}^{M-1}\left(\frac{h}{2}\right)^{2 m} \sum_{s=1}^{M-m}\left(\frac{h}{2}\right)^{2 s} T_{s}\left\{\mathbf{e}_{m}\left(t_{j-1 / 2}\right)\right\}+O\left(h^{2 M+1}\right) \\
= & \sum_{m=1}^{M}\left(\frac{h}{2}\right)^{2 m} T_{m}\left\{\mathbf{y}\left(t_{j-1 / 2}\right)\right\}+O\left(h^{2 M+1}\right),
\end{aligned}
$$


since the last two sums are found to be identical when like powers of $h$ are compared. Thus (4.5) and (4.6c) yield

$$
L_{h}\left[\mathbf{u}_{j}-\mathbf{y}\left(t_{j}\right)-\sum_{m=1}^{M}\left(\frac{h}{2}\right)^{2 m} \mathbf{e}_{m}\left(t_{j}\right)\right]=O\left(h^{2 M+1}\right), \quad j=1,2, \cdots, J .
$$

The boundary conditions in (4.3) and (3.3) yield

$$
\sum_{v=1}^{N} B_{v}\left[\mathbf{u}_{j_{v}}-\mathbf{y}\left(t_{j_{v}}\right)-\sum_{m=1}^{M}\left(\frac{h}{2}\right)^{2 m} \mathbf{e}_{m}\left(t_{j_{v}}\right)\right]=0 .
$$

Now apply the stability result of Lemma 3.3, with

$$
\boldsymbol{\phi}_{j} \equiv\left[\mathbf{u}_{j}-\mathbf{y}\left(t_{j}\right)-\sum_{m=1}^{M}\left(\frac{h}{2}\right)^{2 m} \mathbf{e}_{m}\left(t_{j}\right)\right],
$$

and (4.4) follows. This completes the proof.

It should be pointed out that the residual term in (4.4) is $O\left(h^{2 M+2}\right)$ if the data $A(t)$ and $\mathbf{g}(t)$ are in $P C_{2 M+2}[a, b]$.

There are two general ways in which the asymptotic expansions (4.4) can be employed to justify computing higher order accuracy approximations. First suppose that we can determine net functions $\left\{\mathbf{E}_{m, j}\right\}$ such that on the net

$$
\left\|\mathbf{e}_{m}\left(t_{j}\right)-\mathbf{E}_{m, j}\right\|=O\left(h^{2(M-m)+1}\right), \quad m=1,2, \cdots, M .
$$

Then it clearly follows from (4.4) that

$$
\mathbf{U}_{j}=\mathbf{u}_{j}-\sum_{m=1}^{M}\left(\frac{h}{2}\right)^{2 m} \mathbf{E}_{m, j}
$$

satisfies

$$
\left\|\mathbf{U}_{j}-\mathbf{y}\left(t_{j}\right)\right\|=O\left(h^{2 M+1}\right) .
$$

This is the basis for Fox's difference correction method [2] in which the inhomogeneous terms in (4.2) and (4.3) are approximated by difference quotients (of decreasing order of accuracy as $m$ increases). Then corresponding difference problems are solved for the $\left\{\mathbf{E}_{m, j}\right\}$. There are special difficulties near the endpoints where either external points must be introduced or noncentered differences must be used. A number of recent studies have justified these procedures in special cases [4], [8] and generalized them in various ways [9]. However if, as in the present case, we allow discontinuous data, the endpoint difficulties occur also in the interior. We are not aware of any justification for difference corrections in such cases but it seems clear that this could easily be done.

The other alternative is $h \rightarrow 0$ extrapolation in which there is no difficulty caused by nonsmooth data when the present difference scheme is employed. Specifically we consider a sequence of nets with uniform spacings $h \equiv h_{\mu}>0$ satisfying (3.1), (4.1) and

$$
h_{\mu+1}<h_{\mu}, \quad \mu=0,1,2, \cdots .
$$


Let $t$ be any fixed point common to all the nets specified above. Then on the $\mu$ th net we introduce the integer

$$
j(t, \mu) \equiv \frac{t-t_{0}}{h_{\mu}}
$$

so that $t_{j(t, \mu)}=t$. The corresponding net function satisfying (3.2)-(3.3) on the $\mu$ th net has a value at this net point which we denote by

$$
\mathbf{u}_{j(t, \mu)} \equiv \mathbf{v}(t, \mu) \text {. }
$$

In terms of this notation the asymptotic error estimate (4.4) applied for $j=j(t, \mu)$ on the $\mu$ th net yields

$$
\mathbf{v}(t, \mu)=\mathbf{y}(t)+\sum_{m=1}^{M}\left(\frac{h_{\mu}}{2}\right)^{2 m} \mathbf{e}_{m}(t)+O\left(h_{\mu}^{2 M+1}\right), \quad \mu=0,1,2, \cdots .
$$

With calculations performed on the nets corresponding to $\mu=0,1,2, \cdots, M$ we can obtain approximations which are $O\left(h_{0}^{2 M+1}\right)$ accurate by using iterative interpolation. That is we define

$$
\begin{array}{rlrl}
\Delta_{\mu} \equiv\left(\frac{h_{\mu}}{2}\right)^{2}, & \mu=0,1, \cdots, M, \\
\mathbf{v}(t ; \mu, \mu+1) & \equiv \frac{\Delta_{\mu} \mathbf{v}(t ; \mu+1)-\Delta_{\mu+1} \mathbf{v}(t ; \mu)}{\Delta_{\mu}-\Delta_{\mu+1}}, \mu=0,1, \cdots, M-1, \\
\mathbf{v}(t ; \mu, \mu+1, \mu+2) & \equiv \frac{\Delta_{\mu} \mathbf{v}(t ; \mu+1, \mu+2)-\Delta_{\mu+2} \mathbf{v}(t ; \mu, \mu+1)}{\Delta_{\mu}-\Delta_{\mu+2}}, \\
& : & \mu=0,1, \cdots, M-2 .
\end{array}
$$

It follows from (4.10) that

$$
\begin{aligned}
\mathbf{v}(t ; \mu, \mu+1)-\mathbf{y}(t) & =O\left(h_{\mu}^{4}\right), & & \mu=0,1, \cdots, M-1, \\
\mathbf{v}(t ; \mu, \mu+1, \mu+2)-\mathbf{y}(t) & =O\left(h_{\mu}^{6}\right), & & \mu=0,1, \cdots, M-2,
\end{aligned}
$$

$$
\mathbf{v}(t ; 0,1, \cdots, M)-\mathbf{y}(t)=O\left(h_{0}^{2 M+1}\right) .
$$

The final error will be $O\left(h_{0}^{2 M+2}\right)$, as expected, if the data $A(t)$ and $\mathbf{g}(t)$ are in $P C_{2 M+2}[a, b]$. The scheme (4.11) is just Neville's iterated interpolation; see [3] or $[6$, pp. 258-260]. In actually performing the calculations in (4.11) there may be less danger of cancellation of leading digits and hence less loss in significance if we actually employ the forms

$$
\mathbf{v}(t ; \mu, \mu+1)=\mathbf{v}(t ; \mu+1)+\frac{\Delta_{\mu+1}}{\Delta_{\mu}-\Delta_{\mu+1}}[\mathbf{v}(t ; \mu+1)-\mathbf{v}(t ; \mu)],
$$


and so on. Of course the results (4.12) apply for each point $t$ common to the $M+1$ nets employed. A rather obvious choice for the spacings $h_{\mu}$ is to take $h_{\mu}=\left(\frac{1}{2}\right)^{\mu} h_{0}$. Then at all points on the initial net, with spacing $h_{0}$, the high order accuracy approximations can be determined. More general refinements of the spacing are briefly considered in [3] and in references given there, as are alternative rational fraction extrapolations. (The successive halving indicated above is not the most efficient way to reduce the net spacing.)

4.1. $h \rightarrow 0$ extrapolation with nonuniform nets. In place of the family of uniform nets defined by (4.1) we shall now consider specific sequences of nonuniform nets for which (3.1) is satisfied with no restriction on the points $\tau_{v}$. Let $\left\{t_{j}\right\}$ be any net with, say, $J+1$ points on $[a, b]$ satisfying

$$
\begin{gathered}
t_{0}=a, \quad t_{J}=b, \quad t_{j}>t_{j-1}, \quad j=1,2, \cdots, J, \\
\left\{\tau_{v}\right\} \subset\left\{t_{j}\right\} .
\end{gathered}
$$

We call $\left\{t_{j}\right\}$ a basic net and in terms of it define:

$$
\begin{aligned}
& h_{0} \equiv \max _{1 \leqq j \leqq J}\left(t_{j}-t_{j-1}\right) ; \\
\theta(t) \equiv & \frac{t_{j}-t_{j-1}}{h_{0}}, \quad t_{j-1} \leqq t<t_{j}, \quad j=1,2, \cdots, J .
\end{aligned}
$$

Now we define a sequence of nets $\left\{t_{\mu, j}\right\}, \mu=0,1,2, \cdots$, by

$$
h_{\mu}=2^{-\mu} h_{0}, \quad t_{\mu, 0}=a ;
$$

(4.15b) $t_{\mu, j}=t_{\mu, j-1}+h_{\mu, j}, \quad h_{\mu, j} \equiv \theta\left(t_{\mu, j-1}+0\right) h_{\mu}, \quad j=1,2, \cdots, J_{\mu} \equiv 2^{\mu} J$.

It easily follows that $t_{\mu, J_{\mu}}=b$ and $\left\{\tau_{v}\right\} \subset\left\{t_{\mu, j}\right\}$ for each $\mu=1,2, \cdots$. Note that $\theta(t)$ defined in (4.14) is piecewise constant and satisfies

$$
0<\lambda^{-1} \leqq \theta(t) \leqq 1, \quad t \in[a, b],
$$

where $\lambda \equiv \max _{j, k}\left(t_{j}-t_{j-1}\right) /\left(t_{k}-t_{k-1}\right)$. Thus each net (4.15) satisfies the conditions (3.1). In addition, after the $h_{\mu, j}$ have been defined as in (4.15) we can write

$$
h_{\mu, j}=\theta\left(t_{\mu, j-1}+\frac{1}{2} h_{\mu, j}\right) h_{\mu} .
$$

Finally we point out that the possible points of (jump) discontinuity of $\theta(t)$ are in the basic net $\left\{t_{j}\right\}$ and hence are included in all of the nets (4.15).

Now define in place of (4.3) the principal error functions $\varepsilon_{m}(t)$ as solutions of the multipoint boundary value problems

$$
\begin{gathered}
L \varepsilon_{1}(t)=\theta^{2}(t) T_{1}\{\mathbf{y}(t)\}, \quad \sum_{v=1}^{N} B_{v} \varepsilon_{1}\left(\tau_{v}\right)=0, \\
L \varepsilon_{m}(t)=\theta^{2 m}(t) T_{m}\{\mathbf{y}(t)\}+\sum_{k=1}^{m-1} \theta^{2 k}(t) T_{k}\left\{\varepsilon_{m-k}(t)\right\}, \\
\sum_{v=1}^{N} B_{v} \varepsilon_{m}\left(\tau_{v}\right)=0, \quad m=2,3, \cdots, M .
\end{gathered}
$$


If $\left\{\mathbf{u}_{\mu, j}\right\}$ is the solution of the finite difference boundary value problem (3.2)-(3.3) on the net $\left\{t_{\mu, j}\right\}$ in $(4.15)$, then

$$
\mathbf{u}_{\mu, j}-\mathbf{y}\left(t_{\mu, j}\right)=\sum_{m=1}^{M}\left(\frac{h_{\mu}}{2}\right)^{2 m} \varepsilon_{m}\left(t_{\mu, j}\right)+O\left(h_{\mu}^{2 M+1}\right), j=0,1, \cdots, J_{\mu} .
$$

We assume here that $A(t), \mathbf{g}(t) \in P C_{2 M+1}[a, b]$ and problem (1.1) (1.2) has a unique solution. The proof of the above result is almost exactly that of Theorem 4.1 with appropriate replacements of $h$ by $h_{\mu, j}$ as represented in (4.17), $t_{j}$ by $t_{\mu, j}$ and $t_{j-1 / 2}$ by $t_{\mu, j}-\frac{1}{2} h_{\mu, j}$. However the left-hand side of (4.6c) is now formed as

$$
\sum_{m=1}^{M}\left(\frac{h_{\mu}}{2}\right)^{2 m} L_{h} \varepsilon_{m}\left(t_{\mu, j}\right)
$$

and evaluated using the analogue of (4.6) with (4.18) in place of (4.3).

Since (4.19) is established, as above, for all $\mu=0,1, \cdots$ we can apply iterative interpolation as in (4.11) to obtain higher order accuracy solutions on the basic net $\left\{t_{j}\right\}$, which has arbitrary spacing. Our procedure for nonuniform nets can also be applied to the more general boundary conditions or integral constraints of $\S 5$. The details should be fairly clear and hence are omitted. But we point out that these applications show that $h \rightarrow 0$ extrapolation or Romberg quadrature can be employed to get high order accuracy integral approximations with nonuniform nets and piecewise smooth integrands.

5. Integral constraints. In place of the multipoint boundary conditions (1.2) we consider (1.1) subject to integral constraints for the form

$$
\int_{a}^{b} B(t) \mathbf{y}(t) d t=\boldsymbol{\beta}
$$

Here the rows of the $n \times n$ matrix $B(t)$ are assumed to be linearly independent vectors in $P C_{q}[a, b]$. (We discuss later the more general case of Stieltjes integrals with piecewise continuously differentiable integrators.)

The existence and uniqueness Theorem 2.1 applies to the problem (1.1), (5.1) if, in place of (2.2), we now define

$$
Q \equiv \int_{a}^{b} B(\tau) Y\left(\tau, \tau_{0}\right) d \tau
$$

The sufficiency conditions of Theorem 2.2 employ (2.5b) and

$$
Q_{m} \equiv \int_{a}^{b} B(\tau) Y_{m}\left(\tau, \tau_{0}\right) d \tau, \quad m=0,1, \cdots
$$


Then (5.2) is nonsingular and the $Q_{m}$ for all $m>M$ are nonsingular if $Q_{M}$ is nonsingular and, for some $\lambda<1$,

$$
\begin{aligned}
\int_{a}^{b}\left\|Q_{M}^{-1} B(\tau)\right\|\{\exp & \left.|| \int_{\tau_{0}}^{\tau} k(s) d s \mid\right) \\
& \left.-\left[1+\left|\int_{\tau_{0}}^{\tau} k(s) d s\right|+\cdots+\frac{1}{M !}\left|\int_{\tau_{0}}^{\tau} k(s) d s\right|^{M}\right]\right\} d \tau \leqq \lambda .
\end{aligned}
$$

To approximate the solution of (1.1), (5.1) we use the net (3.1), where now the $\tau_{v}$ include all discontinuity points of $A(t), B(t)$ and $\mathbf{g}(t)$. The difference equations (3.2) are retained and in place of (3.3) we use a trapezoidal rule approximation to $(5.1)$ :

$$
\sum_{j=0}^{J} C_{j} \mathbf{u}_{j}=\boldsymbol{\beta},
$$

with

$$
\begin{aligned}
& C_{0} \equiv \frac{1}{2} h_{1} B\left(t_{0}\right), \quad C_{J} \equiv \frac{1}{2} h_{J} B\left(t_{J}\right), \\
& C_{j} \equiv \frac{1}{2}\left[h_{j} B\left(t_{j}-0\right)+h_{j+1} B\left(t_{j}+0\right)\right], \quad j=1,2 \cdots, J-1 .
\end{aligned}
$$

See (5.16) for alternative coefficients which are simpler in applications.

If we define $S_{0}$ by (3.7b) using the $C_{j}$ above, rather than (3.7a), then as in the proof of Lemma 3.2 we find

$$
S_{0}=\left[\sum_{j=0}^{J} C_{j} Y\left(t_{j}, \tau_{0}\right)\right] Y^{-1}\left(t_{0}, \tau_{0}\right)+O\left(\sum_{j=0}^{J} C_{j} \sigma_{j}\right) .
$$

But since the trapezoidal rule converges for continuous integrands (see proof of Theorem 5.1 below) it follows that, with $Q$ defined in (5.2),

$$
\left\|Q-\sum_{j=0}^{J} C_{j} Y\left(t_{j}, \tau_{0}\right)\right\|
$$

can be made arbitrarily small as $h \rightarrow 0$. Thus, by the Banach lemma, $S_{0}$ is nonsingular provided $Q$ is nonsingular and $h$ is sufficiently small. Lemma 3.2 now gives, in (3.8), the solution of the difference system (3.2), (5.5) provided the modified $S_{j}$ and $C_{j}$ are used and $Q$ in (5.2) is nonsingular.

The stability result (3.13) becomes, under the obvious modified hypothesis of Lemma 3.3,

$$
\left\|\phi_{j}\right\| \leqq K_{1}\left\|\sum_{j=0}^{J} C_{j} \phi_{j}\right\|+K_{3} \max _{1 \leqq i \leqq J}\left\|L_{h} \phi_{i}\right\|, \quad j=0,1, \cdots, J,
$$

where in place of $K_{2}$ we employ

$$
K_{3}=e^{\lambda K^{*}|b-a|}\left[\frac{2}{K}+\frac{K_{1}|b-a|^{2}}{1-\delta_{1}} \max _{a \leqq t \leqq b}\|B(t)\|\right] .
$$

Now we have the following theorem. 
TheOREM 5.1. Let $A(t)$ and $\mathbf{g}(t) \in P C_{p}[a, b]$ and $B(t) \in P C_{q}[a, b]$ for some nonnegative integers $p, q$. Let the generalized boundary value problem (1.1), (5.1) have a unique solution $\mathbf{y}(t)$. Then the difference equations (3.2), (5.5) have a unique solution $\left\{\mathbf{u}_{j}\right\}$ for all nets (3.1) with $h$ sufficiently small and

$$
\left\|\mathbf{y}\left(t_{j}\right)-\mathbf{u}_{j}\right\|= \begin{cases}O\left(h^{2}\right) & \text { if } p \geqq 2, \quad q \geqq 2, \\ O\left(h \omega_{2}(h)\right) & \text { if } p=1, \quad q \geqq 2, \\ O(h) & \text { if } p \geqq 1, \quad q=1, \\ O\left(\omega_{1}(h)+\Omega(h)\right) & \text { if } p=q=0 .\end{cases}
$$

Here $\omega_{v}(h)$ is the modulus of continuity of $\mathbf{y}^{(v)}(t)$ and $\Omega(h)$ that of $B(t) \mathbf{y}(t)$ over any interval of continuity on $[a, b]$.

Proof. From (5.6) with $\phi_{j} \equiv \mathbf{y}\left(t_{j}\right)-\mathbf{u}_{j},(5.1)$ and (5.5) we obtain

$$
\begin{aligned}
\left\|\mathbf{y}\left(t_{k}\right)-\mathbf{u}_{k}\right\| \leqq & K_{1}\left\|\sum_{j=1}^{J} \frac{h_{j}}{2}\left[B\left(t_{j-1}+0\right) \mathbf{y}\left(t_{j-1}\right)+B\left(t_{j}-0\right) \mathbf{y}\left(t_{j}\right)\right]-\int_{t_{j-1}}^{t_{j}} B(t) \mathbf{y}(t) d t\right\| \\
& +K_{3} \max _{1 \leqq i \leqq J}\left\|L_{h} \mathbf{y}\left(t_{i}\right)-L \mathbf{y}\left(t_{i-1 / 2}\right)\right\|, \quad k=0,1, \cdots, J .
\end{aligned}
$$

By Theorem 2.1, or rather its extension to the present case, and the choice of the net it follows that the integrand $B(y) \mathbf{y}(t)$ above has $r \equiv \min (p+1, q)$ continuous derivatives on each interval $\left[t_{j-1}, t_{j}\right]$. Thus if $r \geqq 1$ we can use partial integration (Taylor's theorem) on each such interval to get

$$
\begin{aligned}
E_{j} & \equiv \frac{h_{j}}{2}\left[B\left(t_{j-1}+0\right) \mathbf{y}\left(t_{j-1}\right)+B\left(t_{j}-0\right) \mathbf{y}\left(t_{j}\right)\right]-\int_{t_{j-1}}^{t_{j}} B(t) \mathbf{y}(t) d t \\
& =\int_{-h_{j / 2}}^{h_{j / 2}}\left[B\left(t_{j-1 / 2}+\theta\right) \mathbf{y}\left(t_{j-1 / 2}+\theta\right)\right]^{\prime} \theta d \theta
\end{aligned}
$$

Clearly $\left\|E_{j}\right\| \leqq M_{1}\left(h_{j} / 2\right)^{2}$, where $M_{1}=\frac{1}{2} \max _{\left[t_{j-1}, t_{j}\right]}\left\|[B(t) \mathbf{y}(t)]^{\prime}\right\|$ in the above case. If $r \geqq 2$, so that another integration by parts is valid, we find that $\left\|E_{j}\right\|=O\left(h_{j}^{3}\right)$. If $r=0$, so that the integrand is only continuous, we easily get $\left\|E_{j}\right\|=O\left(h_{j} \Omega\left(h_{j}\right)\right)$, where $\Omega(\delta)$ is the modulus of continuity of $B(t) \mathbf{y}(t)$ on $\left[t_{j-1}, t_{j}\right]$. From these results and the expansions in the proof of Theorem 3.1 the estimates in (5.7) follow. This completes the proof.

To obtain higher order accuracy approximations by $h \rightarrow 0$ extrapolation we again specialize to uniformly spaced nets (for simplicity of presentation only). In analogy with Theorem 4.1 we now have the following theorem.

THEOREM 5.2. For some integer $M \geqq 1$ let $A(t), B(t), \mathbf{g}(t) \in P C_{2 M+1}[a, b]$ and problem (1.1), (5.1) have a unique solution. Let the possible discontinuity points $\left\{\tau_{v}\right\}$ satisfy (3.1) and (4.1) for some sequence of nets with $J=J_{\mu} \rightarrow \infty$. Define $T_{m}\{\phi(t)\}$ as in (4.2) and for $\phi(t) \in P C_{2 M-1}[a, b]$ define the (sum) operator:

$$
S_{m}\{\phi(t)\} \equiv \frac{-2^{2 m}}{(2 m) !} B_{2 m} \int_{a}^{b}[B(t) \phi(t)]^{(2 m-1)} d t, m=1,2, \cdots, M,
$$


where $B_{2 m}$ is the $2 m$-th Bernoulli number. With $\mathbf{y}(t)$ the solution of $(1.1),(5.1)$ define the principal error functions $\mathbf{f}_{m}(t)$ as the solutions of

$$
\begin{aligned}
& L \mathbf{f}_{1}(t)=T_{1}\{\mathbf{y}(t)\}, \\
& \int_{a}^{b} B(t) \mathbf{f}_{1}(t) d t=S_{1}\{\mathbf{y}(t)\} ; \\
& L \mathbf{f}_{m}(t)=T_{m}\{\mathbf{y}(t)\}+\sum_{k=1}^{m-1} T_{k}\left\{\mathbf{f}_{m-k}(t)\right\}, \quad m=1,2, \cdots, M, \\
& \int_{a}^{b} B(t) \mathbf{f}_{m}(t) d t=S_{m}\{\mathbf{y}(t)\}+\sum_{k=1}^{m-1} S_{k}\left\{\mathbf{f}_{m-k}(t)\right\}, \quad m=1,2, \cdots, M .
\end{aligned}
$$

Then $\left\{\mathbf{u}_{j}\right\}$, the solution of the finite difference problem (3.2), (5.5) on the net (3.1), (4.1), satisfies:

$$
\mathbf{u}_{j}-\mathbf{y}\left(t_{j}\right)=\sum_{m=1}^{M}\left(\frac{h}{2}\right)^{2 m} \mathbf{f}_{m}\left(t_{j}\right)+O\left(h^{2 M+1}\right), \quad j=0,1, \cdots, J .
$$

Proof. From the modified form of Theorem 2.1 which applies to problem (1.1), (5.1) it follows that $\mathbf{y}(t) \in P C_{2 M+2}[a, b]$. Thus the development in (4.5) also holds for the present $\mathbf{u}_{j}$ and $\mathbf{y}\left(t_{j}\right)$. We also see that the relations (4.6a) and (4.6b) apply if the $\mathbf{e}_{m}(t)$ are replaced by the $\mathbf{f}_{m}(t)$ defined in (5.9) since only appropriate smoothness properties of these solutions are required and, clearly, $\mathbf{f}_{m}(t) \in P C_{2(M-m)+2}[a, b]$. The result in (4.6c) is also valid with the $\mathbf{e}_{m}(t)$ replaced by the $f_{m}(t)$ since the differential equations in (4.3) and (5.9) are formally identical. Thus we have, exactly as in the derivation of $(4.7 \mathrm{a})$, the relations

$$
L_{h}\left[\mathbf{u}_{j}-\mathbf{y}\left(t_{j}\right)-\sum_{m=1}^{M}\left(\frac{h}{2}\right)^{2 m} \mathbf{f}_{m}\left(t_{j}\right)\right]=O\left(h^{2 M+1}\right), \quad j=1,2, \cdots, J .
$$

From (5.1) and (5.5) we have, employing the Euler-Maclaurin sum formula on $\left[t_{j-1}, t_{j}\right]$, where $B(t) \mathbf{y}(t) \in C_{2 M+1}\left[t_{j-1}, t_{j}\right]$ for $j=1,2, \cdots, J$ :

$$
\begin{aligned}
\sum_{j=0}^{J} C_{j}\left[\mathbf{y}\left(t_{j}\right)-\mathbf{u}_{j}\right] & =\sum_{j=1}^{J}\left\{\frac{h}{2}\left[B\left(t_{j-1}+0\right) \mathbf{y}\left(t_{j-1}\right)+B\left(t_{j}-0\right) \mathbf{y}\left(t_{j}\right)\right]-\int_{t_{j-1}}^{t_{j}} B(t) \mathbf{y}(t) d t\right\} \\
(5.12) & =\sum_{j=1}^{J}\left\{\left.\sum_{m=1}^{M}\left(\frac{h}{2}\right)^{2 m} \frac{2^{2 m}}{(2 m) !} B_{2 m}[B(t) \mathbf{y}(t)]^{(2 m-1)}\right|_{t_{j-1}} ^{t_{j}}+O\left(h^{2 M+2}\right)\right\} \\
& =-\sum_{m=1}^{M}\left(\frac{h}{2}\right)^{2 m} S_{m}\{\mathbf{y}(t)\}+O\left(h^{2 M+1}\right) .
\end{aligned}
$$

In the final step above we have interchanged orders of summation and used the fact that jump discontinuities of $[B(t) \mathbf{y}(t)]^{(m)}$ occur, if at all, at points $\tau_{v}=t_{j_{v}}$ of the net. Similarly from $(5.5 \mathrm{~b})$ and the Euler--Maclaurin formula we obtain, 
since $B(t) \mathbf{f}_{m}(t) \in P C_{2(M-m)+2}[a, b]$ :

$\sum_{j=0}^{J} C_{j} \mathbf{f}_{m}\left(t_{j}\right)=\int_{a}^{b} B(t) \mathbf{f}_{m}(t) d t$

$$
\begin{aligned}
& +\sum_{j=1}^{J}\left\{\frac{h}{2}\left[B\left(t_{j-1}+0\right) \mathbf{f}_{m}\left(t_{j-1}\right)+B\left(t_{j}-0\right) \mathbf{f}_{m}\left(t_{j}\right)\right]-\int_{t_{j-1}}^{t_{j}} B(t) \mathbf{f}_{m}(t) d t\right\}, \\
= & \int_{a}^{b} B(t) \mathbf{f}_{m}(t) d t-\sum_{k=1}^{M-m}\left(\frac{h}{2}\right)^{2 k} S_{k}\left\{\mathbf{f}_{m}(t)\right\}+O\left(h^{2(M-m)+1}\right), \\
& m=1,2, \cdots, M-1 .
\end{aligned}
$$

For $m=M$ we have $B(t) \mathbf{f}_{M}(t) \in P C_{2}[a, b]$ and so

$$
\sum_{j=0}^{J} C_{j} \mathbf{f}_{M}\left(t_{j}\right)=\int_{a}^{b} B(t) \mathbf{f}_{M}(t) d t+O(h) .
$$

Using the integral conditions from (5.9) in (5.13) yields, after rearranging some sums,

$$
\sum_{j=0}^{J} C_{j} \sum_{m=1}^{M}\left(\frac{h}{2}\right)^{2 m} \mathbf{f}_{m}\left(t_{j}\right)=\sum_{m=1}^{M}\left(\frac{h}{2}\right)^{2 m} S_{m}\{\mathbf{y}(t)\}+O\left(h^{2 M+1}\right) .
$$

Thus (5.12) and the above yield

$$
\sum_{j=0}^{J} C_{j}\left[\mathbf{u}_{j}-\mathbf{y}\left(t_{j}\right)-\sum_{m=1}^{M}\left(\frac{h}{2}\right)^{2 m} \mathbf{f}_{m}\left(t_{j}\right)\right]=O\left(h^{2 M+1}\right) .
$$

With $\boldsymbol{\phi}_{j} \equiv\left[\mathbf{u}_{j}-\mathbf{y}\left(t_{j}\right)-\sum_{m=1}^{M}(h / 2)^{2 m} \mathbf{f}_{m}\left(t_{j}\right)\right]$ in the stability result (5.6) we obtain (5.10) by means of (5.11) and (5.14). This completes the proof.

To actually employ this theorem for computing higher order accuracy approximations to the solution of $(1.1),(5.1)$ we proceed as in (4.8)-(4.12) but use the difference problem (3.2), (5.5).

We point out that our problem (1.1), (5.1) could have been replaced by the following two-point boundary value problem:

$$
\begin{aligned}
& \mathbf{y}^{\prime}(t)=A(t) \mathbf{y}(t)+\mathbf{g}(t), \\
& \mathbf{z}^{\prime}(t)=B(t) \mathbf{y}(t), \quad \mathbf{z}(a)=0, \quad \mathbf{z}(b)=\boldsymbol{\beta} .
\end{aligned}
$$

The difference method of $\S 3$ applied to this problem involves systems of order $2 n$, which are avoided in our direct approach. However the "midpoint" scheme applicable to (5.15) avoids the need for special care in treating the discontinuities of $B(t)$ as in (5.5b). This in fact can also be done in our direct treatment by using in $(5.5 \mathrm{a})$, in place of $(5.5 \mathrm{~b})$, the coefficients :

$$
\begin{aligned}
C_{0} & \equiv \frac{1}{2} h_{1} B\left(t_{1-1 / 2}\right), \quad C_{J} \equiv \frac{1}{2} h_{J} B\left(t_{J-1 / 2}\right), \\
C_{j} & \equiv \frac{1}{2}\left[h_{j} B\left(t_{j-1 / 2}\right)+h_{j+1} B\left(t_{j+1 / 2}\right)\right], \quad j=1,2, \cdots, J-1 .
\end{aligned}
$$


We have not employed these coefficients as the analysis becomes rather lengthy since the Euler--Maclaurin formula is no longer relevant. But the error estimates and $h \rightarrow 0$ extrapolation procedure remain valid.

Finally we observe that the procedures and analysis of $\S 3$ and $\S 5$ can easily be combined to treat (1.1) subject to constraints of the form

$$
\sum_{v=1}^{N} B_{v} \mathbf{y}\left(\tau_{v}\right)+\int_{a}^{b} B(t) \mathbf{y}(t) d t=\boldsymbol{\beta} .
$$

In fact the only change in the numerical method is simply to replace the $C_{j}$ by the sum of those $C_{j}$ defined in (5.5b) or (5.16) to those defined in (3.7a). Constraints of the form (5.17) can be represented by Stieltjes integrals with piecewise continuously differentiable integrators. The most general linear constraint applied to continuous $\mathbf{y}(t)$ (i.e., a linear functional on $\left.C_{0}[a, b]\right)$ is given by a Stieltjes integral with integrator of bounded variation. We cannot hope to treat this most general constraint by numerical methods of high order accuracy.

\section{REFERENCES}

[1] E. Coddington And N. Levinson, Theory of Ordinary Differential Equations, McGraw-Hill, New York, 1955.

[2] L. Fox, The Numerical Solution of Two-Point Boundary-Value Problems in Ordinary Differential Equations, Oxford University Press, London, 1957.

[3] W. B. GragG, On extrapolation algorithms for ordinary initial value problems, this Journal, 2 (1965), pp. $384-403$.

[4] P. Henrici, Discrete Variable Methods in Ordinary Differential Equations, John Wiley, New York, 1962.

[5] E. L. INCE, Ordinary Differential Equations, Dover, New York, 1944.

[6] E. IsaAcson and H. B. Keller, Analysis of Numerical Methods, John Wiley, New York, 1966.

[7] H. B. Keller, Numerical Methods for Two-Point Boundary-Value Problems, Blaisdell, Waltham, Massachusetts, 1968.

[8] M. LEES, Discrete methods for nonlinear two-point boundary value problems, Numerical Solution of Partial Differential Equations, J. H. Bramble, ed., Academic Press, New York, 1966, pp. 59-72.

[9] V. L. Pereyra, Highly accurate discrete methods for nonlinear problems, MRC Rep. 749, Mathematics Research Center, U.S. Army, University of Wisconsin, Madison, 1967. 